\title{
Myelitis and cerebral vasculopathy following neck irradiation
}

\author{
B M T P Nawasiwatte ${ }^{1}$, G N N Fernando ${ }^{1}$, D Gunasekera², P S Gunaratne ${ }^{1}$
}

Ceylon Medical Journal 2014; 59: 101-102

\section{Introduction}

Radiation myelitis (RM) is a dreaded complication of radiotherapy (RT) that can manifest years later. The impact of radiation on the spinal cord is determined by the total radiation dose, fraction size and frequency, duration, reirradiation, irradiated cord length and concurrent chemotherapy. The effects of RT are field specific, because they are limited to the intended field of irradiation [1]. We report two patients with progressive RM who subsequently developed unexplained cerebral involvement, raising the possibility of propagation of radiation induced damage beyond the exposure field.

\section{Case 1}

A 62-years old male with laryngeal squamous cell carcinoma underwent RT in 2011. The first phase constituted standard dose of $46 \mathrm{GY}$ in 23 fractions and the second 20 Gy in 10 fractions on a linear accelerator. He subsequently developed cutaneous herpes zoster (HZ) in C3 dermatome twice, which was successfully treated with oral acyclovir.

Eighteen months later he developed weakness of left leg with a right T1 sensory level. The MRI spinal cord showed diffuse increase in signal intensity from cervicomedullary junction to C5/6 inter-vertebral level suggesting RM. MRI brain showed multiple T2 and FLAIR hyperintensities of small vessel disease. There was no evidence of tumour recurrence or metastasis. The limb weakness progressed rapidly making him quadriplegic. During the next four months the MRIs showed progressively increasing signal intensity of the cord extending up to the lower border of T1 vertebra and florid vasculopathy involving the brain and cerebellum.

Figures 1 and 2 showing MR images of patient 1 four months after and eighteen months after RT

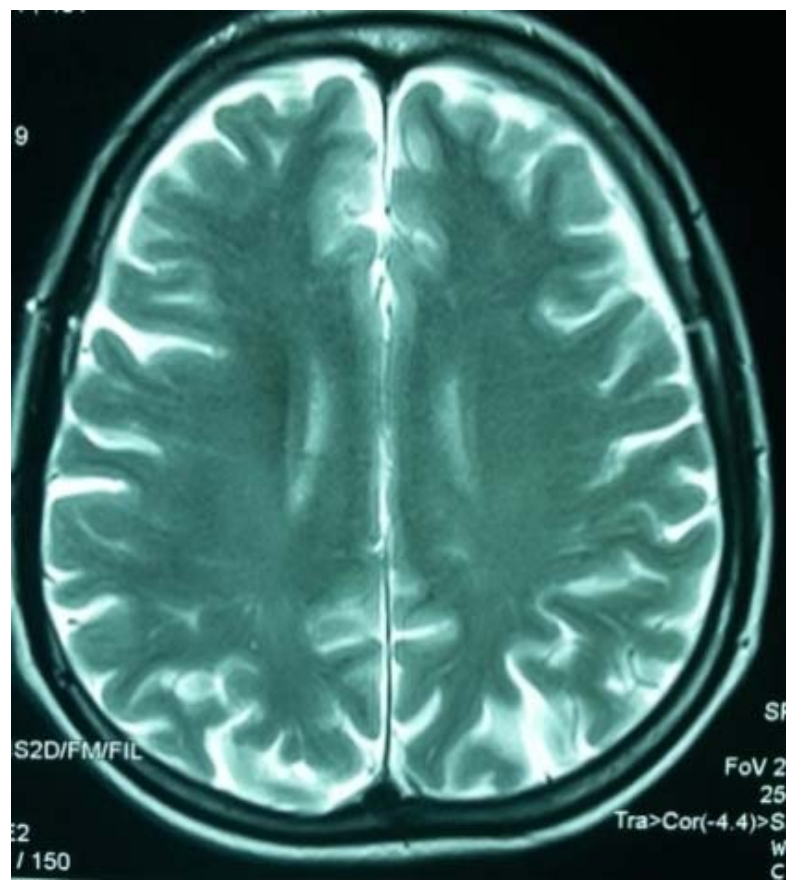

Figure 1.

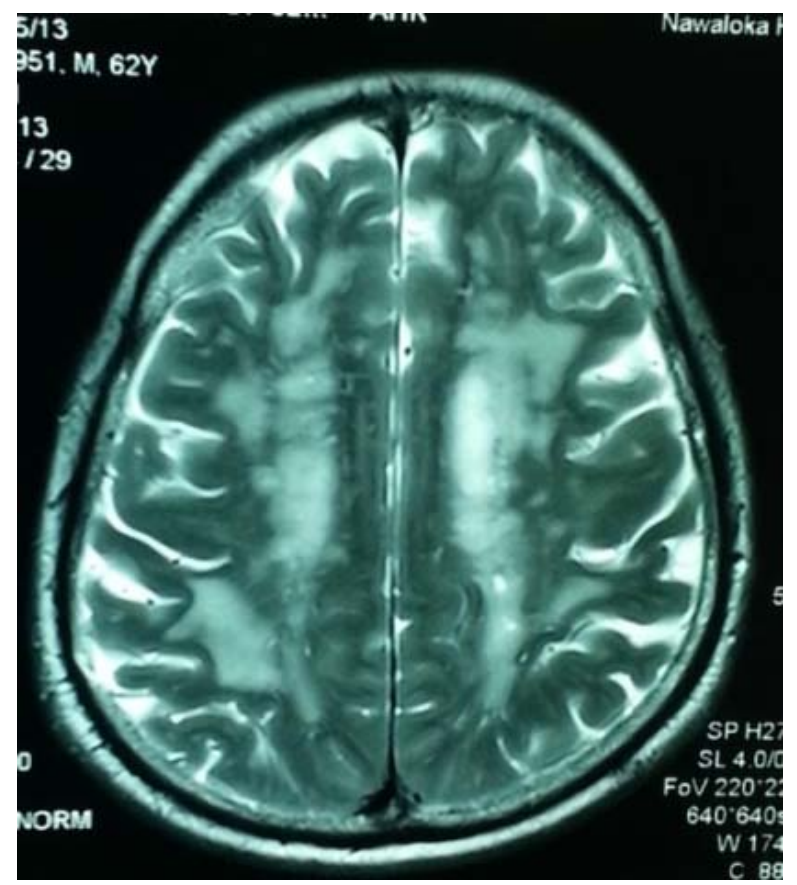

Figure 2.

${ }^{1}$ Neurology Unit 2, National Hospital of Sri Lanka, ${ }^{2}$ National Cancer Institute Maharagama, Sri Lanka.

Correspondence: BMTPN, e-mail: <thusha.nawasiwatte@gmail.com>. Received 2 December 2013 and revised version accepted 21 June 2014. Competing interests: none declared. 
After six months he developed acute confusion and hyponatremia. Despite graded correction, he succumbed to the illness. Basic biochemistry, haematology and infective screening (retroviral, VZV-IgM, CMV, HSV1\&2, mycobacteria, cryptococcal, toxoplasma, VDRL) were negative and CSF analysis was normal.

\section{Case 2}

A 37-years old female with papillary carcinoma underwent thyroidectomy followed by Cobalt-60 teletherapy to the neck and chest, with a total dose of 45 Gy scheduled over 20 fractions. The $\mathrm{I}^{131}$ scan eighteen months later showed residual thyroid tissue and submental nodes. Therefore she was given Radio-iodine (RI) therapy (22.8GBq) as well.

Thirty six months later she developed progressive numbness of legs with a sensory loss up to left T3 level, truncal ataxia and in-coordination of limbs. The MRI showed T2 and FLAIR hyper-intensities involving bilateral pre and post-central gyri and radiation induced changes in cervical and upper thoracic vertebrae. The CT angiogram of brain and spine were normal. She is currently being observed for further progression.

\section{Discussion}

Radiation myelitis is a rare but grave complication of RT. Toxicity of external beam radiotherapy to central nervous system is classified as acute, early-delayed and late-delayed reactions [2]. Delayed radiation myelitis is considered irreversible and typically appears later than six months from exposure as in the case of our patients [2]. For fractions less than 20 Gy given daily up to a total of $55 \mathrm{~Gy}$, the risk of permanent neurologic damage is about $1 \%$ [3]. Although formulae do exist to calculate the relative impact of RT to CNS, those are at best approximations.

Though RM is expected to be limited to the irradiation field, in both these cases RM was followed by cerebral vasculopathy. Direct whole brain irradiation which neither patients received could have caused a similar picture but with more local effects [3]. There was no evidence of atherosclerosis or vasculitis in spinal and cerebral angiograms and biochemical investigations were normal. Therefore there were no other plausible explanation for the intracranial symptoms and MRI changes.

VZV causes multi-focal vasculopathy in immunocompromised patients in whom imaging shows cortical and sub-cortical gray and white matter changes usually restricted to cerebral arterial territories [4]. Patient 1 had a history of dermatomal herpes zoster, but his CSF VZV IgM was negative and MRI changes did not show an arterial territory. There were no other co-morbidities which could explain his cerebral vasculopathy.

$\mathrm{I}^{131}$ can rarely cause RM [5]. Patient 2 received RI, equivalent to about 20-30 Gy of RT, which is within the safety margin for the spinal cord. There was no explanation for her cerebellar signs and the brain gliotic changes on MRI.

Although the effect of radiotherapy are considered field specific these two cases raise the possibility of extended effects of RT on the CNS.

\section{References}

1. Schwartz DL, Schechter GP, Seltzer S, et al. Radiation myelitis following allogenic stem cell transplantation and consolidation radiotherapy for Non Hodgkin's Lymphoma. Journal of Bone Marrow Transplantation 2000; 12: 1355-9.

2. Kadir T, Sarica FB, Ozgur K, Cerinmez M, Nur AM. Delayed radiation myelopathy: Differential diagnosis with positron emission tomography/computed tomography examination. Asian Journal of Neurosurgery 2012; 7: 206-9.

3. Gocheva L. Radiation tolerance of the spinal cord: doctrine, dogmas and data, review article. Archives of Oncology 2000; 8: $131-4$.

4. Amlie-Lefond C, Kleinschmidt-DeMasters BK, Mahalingam $\mathrm{R}$, et al. The vasculopathy of Varicella zoster virus encephalitis. Annals of Neurology 1995; 37: 784-90.

5. Murakami H, Kawahara N, Yahata T, et al. Radiation myelopathy after radioactive iodine therapy for spine metastasis. The British Journal of Radiology 2006; 79: e45-9. 